\title{
Some Steiner Symmetry Results in Overdetermined Boundary Value Problem*
}

\author{
Zhongbo Fang, Anna Wang \\ School of Mathematical Sciences, Ocean University of China, Qingdao, China \\ E-mail: fangzb7777@hotmail.com \\ Received June 17, 2011; revised August 2, 2011; accepted August 10, 2011
}

\begin{abstract}
In this paper, we use the moving planes method to prove that the domain $\Omega$ and the solution $u$ are Steiner symmetric if $u$ is a positive solution of the overdetermined boundary value problem in $\Omega$.
\end{abstract}

Keywords: Steiner Symmetry, Moving Planes Method, Overdetermined Boundary Value Problem

\section{Introduction}

We will present some symmetry results in overdetermined boundary value problem

$$
\begin{gathered}
\Delta u+f(x, u,|\nabla u|)=0 \text { in } \Omega \\
u=0 \quad \text { on } \partial \Omega \\
\frac{\partial u}{\partial v}=c\left(x^{\prime}\right) \text { on } \partial \Omega
\end{gathered}
$$

where $x=\left(x_{1}, x^{\prime}\right) \in R^{n}, \quad x^{\prime}=\left(x_{2}, x_{3}, \cdots, x_{n}\right)$ and $v$ is the outward unit normal to $\partial \Omega$. Here $\Omega$ is a bounded domain of class $C^{2}$ in $R^{n}, f$ is a function of class $C^{1}$ and satisfies certain conditions, $C$ is a differentiable function. Obviously the constant normal derivative of $u$ on $\partial \Omega$ is a special case of this problem.

For the motion of a viscous incompressible fluid moving in straight parallel streamlines through a pipe with planar section $\Omega$ or the torsion of a solid straight bar of given cross section $\Omega$, both of them can be described by the following overdetermined problem

$$
\begin{gathered}
-\Delta u=1 \text { in } \Omega \\
u=0 \text { on } \partial \Omega \\
\frac{\partial u}{\partial v}=\text { const on } \partial \Omega
\end{gathered}
$$

where $\Omega$ is a bounded domain of class $C^{2}$ in $R^{n}$ such that there exists a function $u$ satisfying the above problem, and $v$ is the unit normal to $\partial \Omega$. Using the moving planes method, J. Serrin proved that if the above problem has a solution $u$ in $C^{2}(\bar{\Omega})$, then $\Omega$ must be a ball. For more detail, see [1]. Later there are many au-

${ }^{*}$ The Project-sponsored by SRF for ROCS, SEM. thors who investigated the overdetermined the problem. B. Gidas, W. M. Ni and L. Nirenberg proved symmetry of positive solutions of elliptic equations under the Dirichlet boundary condition via moving planes method,

$$
\begin{aligned}
-\Delta u & =f(u) \text { in } \Omega \\
u & =0 \quad \text { on } \partial \Omega
\end{aligned}
$$

See [2]. The paper [3] extended the result of [2], but it did not contain the gradient. A. Colesanti considered the positive solutions of a more general p-Laplacian equation under the overdetermined conditions which has only one critical point via moving planes method too,

$$
\begin{gathered}
\operatorname{div}\left((|\nabla u|)^{p-2} \Delta u\right)+f(u,|\nabla u|)=0 \text { in } \Omega \backslash\{P\} \\
u=0 \text { on } \partial \Omega \\
\frac{\partial u}{\partial v}=c>0 \text { on } \partial \Omega .
\end{gathered}
$$

See [4]. Recently there have been found several other new approaches in studying symmetry problem, such as continuous Steiner symmetrization, domain derivatives and some geometry method, see [5-9]. The moving planes method is a classical technique and is very useful in dealing with symmetry problems, so we still use it to extend the results of $[2,3]$ to the overdetermined boundary problem containing gradient.

We shall prove that $\Omega$ and $u$ are Steiner symmetric with respect to the hyperplane $\left\{x_{1}=\lambda\right\}$ for some real number $\lambda$. To be more specifically, a set $A$ is Steiner symmetric with respect to the hyperplane $\left\{x_{1}=\mu\right\}$ if it is symmetric with respect to the hyperplane $\left\{x_{1}=\mu\right\}$ and convex in the $x_{1}$ direction. The 
function $u$ is called Steiner symmetric with respect to the hyperplane $\left\{x_{1}=\mu\right\}$, we mean that the level sets of $u$ are Steiner symmetric with respect to the hyperplane $\left\{x_{1}=\mu\right\}$. Throughout this paper, we consider $\Omega$ as a bounded domain of class $C^{2}$ in $R^{n}$ unless otherwise stated.

Section 2 of this paper is devoted to the preliminary results. In Section 3, we will present our main results and proofs.

\section{Preliminary Results}

In this section we will introduce the notations in the moving planes method and four results.

Let $\mu$ be a real number, $\Omega$ is a bounded domain in $R^{n}$ with a smooth boundary $\partial \Omega, \lambda_{1} \equiv \max \left\{x_{1} \mid x \in \bar{\Omega}\right\}$. We choose a hyperplane perpendicular to the $x_{1}$ direction in the process of moving a plane.

Consider the hyperplane $T_{\mu}:=\left\{x \in R^{n} \mid x_{1}=\mu\right\}$. For sufficiently large positive number $\mu . T_{\mu}$ is disjoint from $\Omega$. As we decrease $\mu$, at some moment, $T_{\mu}$ begins to intersect $\Omega$ and from that moment $T_{\mu}$ will cut off from $\Omega$ an open cap $\Sigma_{\mu}=\left\{x \in \Omega \mid x_{1}>\mu\right\}$. Let $\Sigma_{\mu}^{\prime}$ denote the reflection of $\Sigma_{\mu}$ across the plane $T_{\mu}$. At the beginning of the process, $\Sigma_{\mu}^{\prime}$ will be contained in $\Omega$, until one of the following events occurs:

A1) $\Sigma_{\mu}^{\prime}$ becomes internally tangent to $\partial \Omega$ at some point $P$ not on $T_{\mu}$,

A2) $T_{\mu}$ reaches a position where it is orthogonal to $\partial \Omega$ at some point $Q$.

We denote the hyperplane $T_{\mu}$ when it reaches either one of these positions by $T_{\lambda}$ and we call $\Sigma_{\lambda}$ (an open cap by $T_{\lambda}$ ) the maximal cap. Clearly its reflection $\Sigma_{\mu}^{\prime}$ is contained in $\Omega$.

Throughout this paper, the following notations will be used: $u_{i}=\frac{\partial u}{\partial x_{i}}, u_{i j}=\frac{\partial^{2} u}{\partial x_{i} \partial x_{j}}$.

The following two lemmas are due to J. Serrin. We will present the lemma, for detailed proof, see [8].

Lemma 2.1 Let $\Omega$ be a domain with $C^{2}$ boundary and $T$ be a hyperplane containing the normal to $\partial \Omega$ at some point $Q$. Let $\Omega^{*}$ denote the portion of $\Omega$ lying on some particular side of $T$.

Suppose that $w$ is of class $C^{2}$ in the closure of $\Omega^{*}$ and satisfies the elliptic inequality

$$
L w=\sum_{i, j=1}^{n} a_{i j}(x) w_{i j}+\sum_{i=1}^{n} b_{i}(x) w_{i} \leq 0, x \in \Omega^{*}
$$

where the coefficients are uniformly bounded. We assume that the matrix $a_{i j}$ is uniformly definite

$$
\sum_{i, j=1}^{n} a_{i j}(x) \xi_{i} \xi_{j} \geq k|\xi|^{2}, k=\text { const }>0,
$$

and that

$$
\sum_{i, j=1}^{n}\left|a_{i j} \xi_{i} \eta_{j}\right| \leq K(|\xi \cdot \eta|+|\xi| \cdot|d|), K=\text { const }>0,
$$

where $\xi=\left(\xi_{1}, \cdots, \xi_{n}\right)$ is an arbitrary real vector,

$\eta=\left(\eta_{1}, \cdots, \eta_{n}\right)$ is the unit normal to the plane $T$, and $d$ is the distance from $T$. Suppose also $w \geq 0$ in $\Omega^{*}$ and $w=0$ at $Q$. Let $s$ be any direction at $Q$ which enters $\Omega^{*}$ nontangentially. Then

$$
\frac{\partial w}{\partial s}>0 \text { or } \frac{\partial^{2} w}{\partial s^{2}}>0 \text { at } Q,
$$

unless $w \equiv 0$.

Let $\Omega$ be a domain in $R^{n}, u \in C^{2}(\bar{\Omega})$ and $u \neq 0$. Consider a linear differential operator of second order of the form

$$
L u \equiv \sum_{i, j=1}^{n} a_{i j}(x) u_{i j}+\sum_{i=1}^{n} b_{i}(x) u_{i}+c(x) u
$$

where $L$ is uniformly elliptic and the coefficients of $L$ are bounded. Here and in what follows, we use summation convention.

The following lemma is a refinement of Hopf's boundary point lemma (See [5]).

Lemma 2.2 Under the above assumptions, suppose that $L u \leq 0$ and $u \geq 0$ in $\Omega$,

1) If $x_{0} \in \partial \Omega, u\left(x_{0}\right)=0$ and $\Omega$ satisfies an interior ball conditions at $x_{0}$, then we have

$$
\frac{\partial u}{\partial v}\left(x_{0}\right)<0
$$

where the vector $v=\left(v_{1}, \cdots, v_{n}\right)$ denotes the outer (unit normal) to $\partial \Omega$ at $x_{0}$.

2) $u \geq 0$ in $\Omega$.

Lemma 2.2 is well known for us in case $c(x)<0$. Here, we note the fact that it holds without the restriction $c<0$.

Now we will present another key proposition which describes the property near the boundary.

Proposition 2.3 Let $\Omega$ be a bounded domain of class $C^{2}$ in $R^{n}$. Let $f$ be of class $C^{1}$. Assume that $u \in C^{2}(\Omega)$ is a solution of the problem

$$
\begin{gathered}
-\Delta u=f(u,|\nabla u|) \text { in } \Omega, \\
u>0 \text { in } \Omega, \\
u=0 \text { on } \partial \Omega .
\end{gathered}
$$

If $\lambda$ is a real number which defines the maximal cap, then for each point $x_{0} \in \partial \Omega \bigcap\left\{x_{1}>\lambda\right\}$, it holds either $u_{1}\left(x_{0}\right)<0$ or else $u_{11}\left(x_{0}\right)>0$, that is, $u$ is a strictly decreasing function of $x_{1}$ near $x_{0}$.

Proof. Note that $v_{1}>0$ at $x_{0}$. By the hypothesis, it 
is obvious that $\frac{\partial u}{\partial v} \leq 0$ on $\partial \Omega \bigcap\left\{x_{1}>\lambda\right\}$, and so $u_{1} \leq 0$ on $\partial \Omega \cap\left\{x_{1}>\lambda\right\}$.

First, suppose $f(0,0) \geq 0$. Then we get

$$
\begin{aligned}
0 & =\Delta u+f(u,|\nabla u|) \\
& =\Delta u+f(u,|\nabla u|)-f(0,0)+f(0,0) \\
& \geq \Delta u+f(u,|\nabla u|)-f(0,0)
\end{aligned}
$$

Denote

$$
\begin{gathered}
p=\left(p_{1}, p_{2}, \cdots, p_{n}\right)=\left(u_{x_{1}}, u_{x_{2}}, \cdots, u_{x_{n}}\right), \\
f[u] \equiv f(u,|\nabla u|) .
\end{gathered}
$$

By the mean value theorem of multidimensional calculus,

$$
f[u]-f[0]=\sum_{i=1}^{n} \frac{\partial f}{\partial p_{i}} .
$$

So for certain bounded functions $b_{i}(x), c(x)$,

$$
\begin{aligned}
0 & \geq \Delta u+f(u,|\nabla u|)-f(0,0) \\
& =\Delta u+\sum_{i=1}^{n} b_{i}(x) u_{i}+c(x) .
\end{aligned}
$$

Thus it follows that $\frac{\partial u}{\partial v}\left(x_{0}\right)<0$ by Lemma 2.2. Since $\nabla u$ is the opposite direction to the outward normal $v$ on $\partial \Omega$ and $v_{1}>0$ at $x_{0}$, we conclude $u_{1}\left(x_{0}\right)<0$.

Now, suppose $f(0,0)<0$ and $u_{1}\left(x_{0}\right)=0$ and $\Delta u\left(x_{0}\right)=-f(0,0)$ by the hypotheses. We claim

$$
u_{i j}\left(x_{0}\right)=-f(0,0) v_{i} v_{j} \text { for each } 1 \leq i, j \leq n
$$

Since (2.1) is invariant under a rotation of coordinate axes, we may consider a coordinate frame with origin at $x_{0}$, the $x_{1}$ axis being directed along the outward normal to $\partial \Omega$ at $x_{0}$ (at this moment, $v=(1,0, \cdots, 0)$ ). In this frame we can represent the boundary of $\Omega$ locally by the equation

$$
x_{1}=\varphi\left(x_{2}, \cdots, x_{n}\right), \phi \in C^{2} .
$$

Since $u=0$ on $\partial \Omega$, we have

$$
u\left(\phi, x_{2}, \cdots, x_{n}\right)=0
$$

Differentiating (2.2) with respect to $x_{i}(i=2, \cdots, n)$, we obtain

$$
u_{1} \phi_{i}+u_{i}=0
$$

Differentiating (2.3) with respect to $x_{j}(j=2, \cdots, n)$, we obtain

$$
u_{i j}+u_{i 1} \phi_{j}+\left(u_{1 j}+u_{11} \phi_{j}\right) \phi_{i}+u_{1} \phi_{i j}=0
$$

Evaluating (2.4) at $x_{0}$, where $\phi_{i}=0$ and $u_{1}=0$, we find

$$
u_{i j}\left(x_{0}\right)=0 \text { for } 2 \leq i, j \leq n .
$$

Thus $u_{11}\left(x_{0}\right)=-f(0,0)$. Since $u_{1} \leq 0$ on $\partial \Omega \cap\left\{x_{1}>\lambda\right\}$ and $u_{1}\left(x_{0}\right)=0$, the mapping $\left(x_{2}, \cdots, x_{n}\right) \mapsto u_{1}\left(\phi, x_{2}, \cdots, x_{n}\right)$ has a maximum at $x_{0}$. Hence $u_{1 i}\left(x_{0}\right)=0$ for $2 \leq i \leq n$. So far we have showed that $(2.1)$ holds for $v=(1,0, \cdots, 0)$. Returning to the original coordinate axes, we can see that (2.1) holds for each $1 \leq i, j \leq n$. Since $f(0,0)<0$ and $v_{1}>0$, we conclude $u_{11}\left(x_{0}\right)>0$.

Proposition 2.3 is proved.

Using the same argument, we obtain the following corollary immediately.

Corollary 2.4 Let $\Omega$ be a bounded domain of class $C^{2}$ in $R^{n}$. Let $f$ be of class $C^{1}$. Assume that $u \in C^{2}(\Omega)$ is a solution of the problem

$$
\begin{gathered}
-\Delta u=f(|\nabla u|) \text { in } \Omega, \\
u>0 \text { in } \Omega, \\
u=0 \text { on } \partial \Omega .
\end{gathered}
$$

If $\lambda$ is a real number which defines the maximal cap, then for each point $x_{0} \in \partial \Omega \bigcap\left\{x_{1}>\lambda\right\}$, it holds either $u_{1}\left(x_{0}\right)<0$ or else $u_{11}\left(x_{0}\right)>0$, that is, $u$ is a strictly decreasing function of $x_{1}$ near $x_{0}$.

Remark: In the Proposition 2.3, the $C^{1}$ hypothesis on $f$ can be replaced by either of the following conditions:

1) $f(|\nabla u|)=f_{1}(|\nabla u|)+f_{2}(|\nabla u|)$ for all $u$, where $f_{1} \in C^{1}$ and $f_{2}$ is nondecreasing,

2) $f$ is locally Lipschitz continuous.

\section{Main Results and Proofs}

Now we will present our main results.

Theorem 3.1 Let $f$ be a continuous function defined on $R^{n+1}$ and satisfies the following conditions:

1) $f$ is symmetric in $x_{1}$ and nonincreasing in $x_{1}$ for $x_{1}>0$,

2) $f_{u}$ is continuous for $x \in \Omega$.

If there exists a positive solution $u$ in $u \in C^{2}(\bar{\Omega})$ satisfying

$$
\begin{gathered}
-\Delta u=f(x, u,|\nabla u|) \quad \text { in } \Omega, \\
u=0 \quad \text { on } \partial \Omega, \\
\frac{\partial u}{\partial v}=c\left(x^{\prime}\right) \quad \text { on } \partial \Omega .
\end{gathered}
$$

Then we have

1) There exists a real number $\lambda$ such that $\Omega$ and $u$ are Steiner symmetric with respect to the hyperplane 
$\left\{x_{1}=\lambda\right\}$.

2) For each $x \in \partial \Omega, v_{1}(x)=0$ implies that $x_{1}=\lambda$, and there exists a $C^{2}$ function $\varphi: \Omega^{\prime} \rightarrow R$ such that $\Omega=\left\{\left(x_{1}, x^{\prime}\right) \mid 2 \lambda-\varphi\left(x^{\prime}\right)<x_{1}<\varphi\left(x^{\prime}\right)\right\}$, where $\Omega^{\prime}=\Omega \bigcap\left\{x_{1}=\lambda\right\}$.

3) In the case when $\lambda$ is not zero, $f$ is independent of the variable $x_{1}$ in $\Omega$.

Proof. For each real number $\alpha$, where $\lambda \leq \alpha \leq \lambda_{1}$, we define functions $v$ and $w$ in $\Sigma_{\alpha}$ :

$$
v(x)=u\left(x^{\alpha}\right) \text { and } w=v-u .
$$

Then $v$ and $w$ satisfy, respectively

$$
\begin{gathered}
-\Delta v=f(x, v,|\nabla v|), x \in \Sigma_{\alpha}, \\
v=u, x \in \partial \Sigma_{\alpha} \cap T_{\alpha}, \\
v \geq 0, x \in \partial \Sigma_{\alpha} \cap T_{\alpha}^{c} .
\end{gathered}
$$

and

$$
\begin{aligned}
& -\Delta w=f\left(x^{\alpha}, v,|\nabla v|\right)-f(x, u,|\nabla u|), x \in \Sigma_{\alpha} \\
& w \geq 0, x \in \partial \Sigma_{\alpha}
\end{aligned}
$$

By the mean value theorem, (3.1) can be rewritten into

$$
\sum_{i, j=1}^{n} a_{i j}(x) w_{i j}+\sum_{i=1}^{n} b_{i}(x) w_{i}+c(x)=0,
$$

where $a_{i j}, b_{i}$ and $c$ are certain bounded functions. Now we claim that for each real number $\alpha\left(\lambda<\alpha<\lambda_{1}\right)$,

$$
v(x)>u(x) \text { in } \Sigma_{\alpha},
$$

It follows from Proposition 2.3 that (3.2) holds for each $\alpha$ sufficiently close to $\lambda_{1}$.

Let $\beta \equiv \inf \left\{\lambda \leq \alpha \mid(3.2)\right.$ holds for each $\left.\alpha<\lambda_{1}\right\}$. We will prove that $\beta=\lambda$. Assume $\beta>\lambda$. For any point $x_{0} \in \partial \Sigma_{\beta} \backslash T_{\beta}$, we have $x_{0}^{\beta} \in \Omega$. So we find $0=u\left(x_{0}\right)<u\left(x_{0}^{\beta}\right)$.

Hence $w \neq 0$ in $\Sigma_{\beta}$. Since $w \geq 0$ in $\Sigma_{\beta}$, applying Lemma 2.2 to $\Omega \equiv \Sigma_{\beta}$, we obtain

$$
w>0, x \in \Sigma_{\beta} \text {, and } w_{1}>0, x \in T_{\beta} \cap \Omega .
$$

So we find $v(x)>u(x)$ in $\Sigma_{\beta-\varepsilon}$ for sufficiently small $\varepsilon>0$.

We reach a contradiction with the choice of $\beta$. Hence $\beta=\lambda$ and this implies that

$$
v(x)>u(x) \text { in } \Sigma_{\alpha} \text { for all } \alpha>\lambda,
$$

and

$$
v(x) \geq u(x) \text { in } \Sigma_{\lambda} .
$$

By Lemma 2.2, we see that either of the following facts holds:

B1) $w>0$ at all interior points of $\Sigma_{\lambda}$, or else

B2) $w \equiv 0$ in $\Sigma_{\lambda}$.
Now, assume that $w \neq 0$ in $\Sigma_{\lambda}$, i.e., (B1) holds.

In the case (A1), by Lemma 2.2, we see

$$
\frac{\partial w}{\partial v}\left(P^{\lambda}\right)<0,
$$

where $P^{\lambda}$ is the reflected value of $P$ across $T_{\lambda}$. But this is a contradiction, since $\frac{\partial u}{\partial v}\left(P^{\lambda}\right)=\frac{\partial v}{\partial v}\left(P^{\lambda}\right)$. In the case (A2), since we cannot apply Lemma 2.2 to the function $w$, we make use of the substitution for $w$. Let $\tilde{w}=w \mathrm{e}^{k x_{1}}$, where $k$ is a constant to be determined. Then we obtain

$$
\tilde{w} \geq 0 \text { in } \Sigma_{\lambda}, \quad \tilde{w}=0 \text { at } Q
$$

and

$$
\sum_{i, j=1}^{n} a_{i j}(x) \tilde{w}_{i j}+\sum_{i=1}^{n} \tilde{b}_{i}(x) \tilde{w}_{i}+\tilde{c} x=0
$$

where $\tilde{b}_{i}=b_{i}-2 k a_{1 i}, \quad \tilde{c}=a_{11} k^{2}-b_{1} k+c$.

For sufficiently large $k$, we have

$$
\sum_{i, j=1}^{n} a_{i j}(x) \tilde{w}_{i j}+\sum_{i=1}^{n} \tilde{b}_{i}(x) \tilde{w}_{i}+\tilde{c} x \leq 0 \text { in } \Sigma_{\lambda} .
$$

By Lemma 2.1, it follows that

$$
\frac{\partial \tilde{w}}{\partial s}>0 \text { or } \frac{\partial^{2} \tilde{w}}{\partial s^{2}}>0 \text { at } Q .
$$

At the point $Q$, since $\tilde{w}=0$, we find

$$
\frac{\partial w}{\partial s}=\frac{\partial \tilde{w}}{\partial s} \mathrm{e}^{-k x_{1}} \text { and } \frac{\partial^{2} w}{\partial s^{2}}=\left(\frac{\partial^{2} \tilde{w}}{\partial s^{2}}-2 k s_{1} \frac{\partial \tilde{w}}{\partial s}\right) \mathrm{e}^{-k x_{1}} .
$$

Thus

$$
\frac{\partial w}{\partial s}>0 \text { or } \frac{\partial^{2} w}{\partial s^{2}}>0 \text { at } Q .
$$

So far we have already showed that (B1) is impossible. And obviously (B2) implies that $u$ is symmetric with respect to $T, \Omega$ is Steiner symmetric with respect to $T$. Meanwhile, $w>0$ in $\Sigma_{\alpha}$ for all $\alpha \geq \lambda, u$ is symmetrically decreasing in $x_{1}$. This completes the proof of (1).

By the symmetry of a domain $\Omega$ and the definition of the maximal cap, for each $x \in \partial \Omega, v_{1}(x)=0$ implies $x_{1}=\lambda$. So $x_{1}>\lambda$ implies $v_{1}(x)>0$. So we can see from the implicit function theorem that there exists a $C^{2}$ function $\varphi: \Omega^{\prime} \rightarrow R$ such that $\Omega=\left\{\left(x_{1}, x^{\prime}\right) \mid 2 \lambda-\varphi\left(x^{\prime}\right)<x_{1}<\phi\left(x^{\prime}\right)\right\}$. Finally, the symmetry of $u$ implies $\Delta u\left(x_{1}, x^{\prime}\right)=\Delta u\left(2 \lambda-x_{1}, x^{\prime}\right)$ in $\Omega$. Thus $f$ is symmetric with respect to the plane $\left\{x_{1}=\lambda\right\}$ in $\Omega$. If $\lambda \neq 0$, by the hypothesis of $f$, we can see that $f$ is independent of the variable $x_{1}$ in $\Omega$. This completes the proof of the theorem.

Using Lemma 2.1, Lemma 2.2, Proposition 2.3, Cor- 
ollary 2.4 and still the same argument, we can obtain the following two corollaries.

Corollary 3.2 Let $f$ be a continuous function defined on $R^{n+1}$ and satisfies the following conditions:

1) $f$ is symmetric in $x_{1}$ and nonincreasing in $x_{1}$ for $x_{1}>0$,

2) $f_{u_{i}}$ is continuous for $x \in \Omega$. ing

If there exists a positive solution $u \in C^{2}(\bar{\Omega})$ satisfy-

$$
\begin{gathered}
-\Delta u=f(x,|\nabla u|) \quad \text { in } \Omega, \\
u=0 \quad \text { on } \partial \Omega, \\
\frac{\partial u}{\partial v}=c\left(x^{\prime}\right) \quad \text { on } \partial \Omega .
\end{gathered}
$$

Then we have

1) There exists a real number $\lambda$ such that $\Omega$ and $u$ are Steiner symmetric with respect to the hyperplane $\left\{x_{1}=\lambda\right\}$.

2) For each $x \in \partial \Omega, v_{1}(x)=0$ implies that $\left\{x_{1}=\lambda\right\}$, and there exists a $C^{2}$ function $\varphi: \Omega^{\prime} \rightarrow R$ such that $\Omega=\left\{\left(x_{1}, x^{\prime}\right) \mid 2 \lambda-\varphi\left(x^{\prime}\right)<x_{1}<\varphi\left(x^{\prime}\right)\right\}$, where $\Omega^{\prime}=\Omega \cap\left\{x_{1}=\lambda\right\}$.

3) In the case when $\lambda$ is not zero, $f$ is independent of the variable $x_{1}$ in $\Omega$.

Corollary 3.3 Let $f$ be a function of class $C^{1}$ defined on $R$. If there exists a positive solution $u \in C^{2}(\bar{\Omega})$ satisfying

$$
\begin{gathered}
-\Delta u=f(|\nabla u|) \quad \text { in } \Omega, \\
u=0 \quad \text { on } \partial \Omega, \\
\frac{\partial u}{\partial v}=c\left(x^{\prime}\right) \quad \text { on } \partial \Omega .
\end{gathered}
$$

Then we have

1) There exists a real number $\lambda$ such that $\Omega$ and $u$ are Steiner symmetric with respect to the hyperplane $\left\{x_{1}=\lambda\right\}$.

2) For each $x \in \partial \Omega, v_{1}(x)=0$ implies that $\left\{x_{1}=\lambda\right\}$, and there exists a $C^{2}$ function $\varphi: \Omega^{\prime} \rightarrow R$ such that

$$
\begin{gathered}
\Omega=\left\{\left(x_{1}, x^{\prime}\right) \mid 2 \lambda-\varphi\left(x^{\prime}\right)<x_{1}<\varphi\left(x^{\prime}\right)\right\}, \text { where } \\
\Omega^{\prime}=\Omega \bigcap\left\{x_{1}=\lambda\right\} .
\end{gathered}
$$

\section{References}

[1] J. Serrin, "A Symmetry Problem in Potential Theory," Archive for Rational Mechanics and Analysis, Vol. 43, No. 4, 1971, pp. 304-318. doi:10.1007/BF00250468

[2] B. Gidas, W. M. Ni and L. Nirenberg, "Symmetry and Related Properties via the Maximum Principle," Communications in Mathematical Physics, Vol. 68, No. 3, 1979, pp. 209-243. doi:10.1007/BF01221125

[3] C. H. Kim, "Steiner Symmetry in Overdetermined Boundary Value Problems," Doctor's Degree Thesis, Chonnam National University, Kwangju, Korea, 2002.

[4] A. Colesanti, "A Symmetry Result for the p-Laplacian Equation via the Moving Planes Method," Applicable Analysis, Vol. 55, No. 3-4, 1994, pp. 207-213.

[5] F. Brock and A. Henrot, "A Symmetry Result for an Overdetermined Elliptic Problem Using Continuous Rearrangement and Domain Derivative," Rendiconti del Circolo Matematico di Palermo, Vol. 51, No. 3, 2002, pp. 375-390. doi:10.1007/BF02871848

[6] M. Choulli and A. Henrot, "Use of the Domain Derivative to Prove Symmetry Results in Partial Differential Equations," Mathematische Nachrichten, Vol. 192, 1998, pp. 91-103. doi:10.1002/mana.19981920106

[7] I. Fragala, I. F. Gazzaola and B. Kawohl, "Overdetemined Boundary Value Problems with Possibly Degenerate Ellipticity: A Geometry Approach," Mathematische Zeitschrift, Vol. 254, No. 1, 2006, pp. 117-132. doi:10.1007/s00209-006-0937-7

[8] N. Garofalo and J. Lewis, "A Symmetry Result Related to Some Overdeternined Boundary Value Problems," American Journal of Mathematics, Vol. 111, No. 1, 1989, pp. 9-33. doi: $10.2307 / 2374477$

[9] L. Ragous, "Symmetry Theorems via the Continuous Steiner Symmetrization," Electronic Journal of Differential Equations, Vol. 2000, No. 44, 2000, pp. 1-11. 